\section{Aussichtsreicher immuntherapeutischer Ansatz}

Seit einiger Zeit befinden sich verschiedene immuntherapeutische Ansätze für die Behandlung des KopfHals-Karzinoms in klinischer Entwicklung. Um die körpereigene Abwehr gegen Kopf-Hals-Karzinome aktivieren zu können, ist eine genauere Charakterisierung der tumorinfiltrierenden Lymphozyten (TIL) hilfreich.

$\mathrm{H}$ PV-induzierte Plattenepithelkarzinome des Kopf-HalsBereiches - die fast ausschließlich den Oropharynx betreffen - befinden sich zum Zeitpunkt der Diagnosestellung häufig schon in einem fortgeschrittenen Stadium; dennoch ist die Prognose hier besser als bei den mit Tabak- und Alkoholkonsum assoziierten Tumoren. Diverse Befunde bei HPV-positiven Tumoren, wie z. B. eine ausgeprägte Infiltration mit TZellen, deuten auf eine verstärkte lokale tumorspezifische TZell-Antwort hin.

In einer explorativen Studie wurde Tumorgewebe von $50 \mathrm{~Pa}$ tienten auf das Vorhandensein von HPV sowie auf Überexpression von p53 - eines der häufigsten immunhistochemischen Merkmale der nicht-viral bedingten Kopf-Hals-Karzinome - getestet. Fast alle zwölf HPV16-positiven Tumoren befanden sich im Oropharynx.

Es gelang, T-Lymphozyten aus Tumorbiopsaten von $19 \mathrm{~Pa}-$ tienten zu kultivieren. Sechs von acht der HPV-positiven Tumoren wiesen HPV16-spezifische T-Zellen auf, dagegen keiner der zwölf HPV-negativen Tumoren. Funktionell aktive HPVspezifische T-Zellen konnten im Tumor bzw. in Lymphknoten entdeckt werden. Die lokale HPV-spezifische T-Zell-Antwort umfasste eine gemischte Population von TH1-, TH2- und regulatorischen T-Zellen sowie von IFN-gamma-produzierenden CD8-positiven T-Zellen. Möglicherweise spielen die lokalen adaptiven Immunzellen eine Rolle beim besseren Ansprechen auf Chemotherapie sowie dem längeren Überleben dieser Patientengruppe.

Die Autoren halten es für möglich, dass die Stimulation der lokalen Immunität helfen könnte, die Balance in Richtung einer feindlichen, tumoriziden Umgebung zu verändern, zumal der Oropharynx auch relativ gut zugänglich ist. Man könnte ähnliche Strategien wie bei HPV16-induzierten Läsionen der Vulva einsetzen, wo man bereits mit verschiedenen Formen von Immuntherapie erfolgreich experimentiert.

Fazit: Die gegen Virus-Peptide gerichtete spezifische T-ZellImmunität verweist den Autoren zufolge auf eine Anti-TumorAktivität. Dies spricht dafür, die Arbeiten an einer immuntherapeutischen Option voranzutreiben.

Waltraud Paukstadt

Heusinkveld $\mathrm{M}$ et al. Systemic and local human papillomavirus 16-specific T-cell immunity in patients with head and neck cancer. Int J Cancer. 2012;131(2):E74-85.

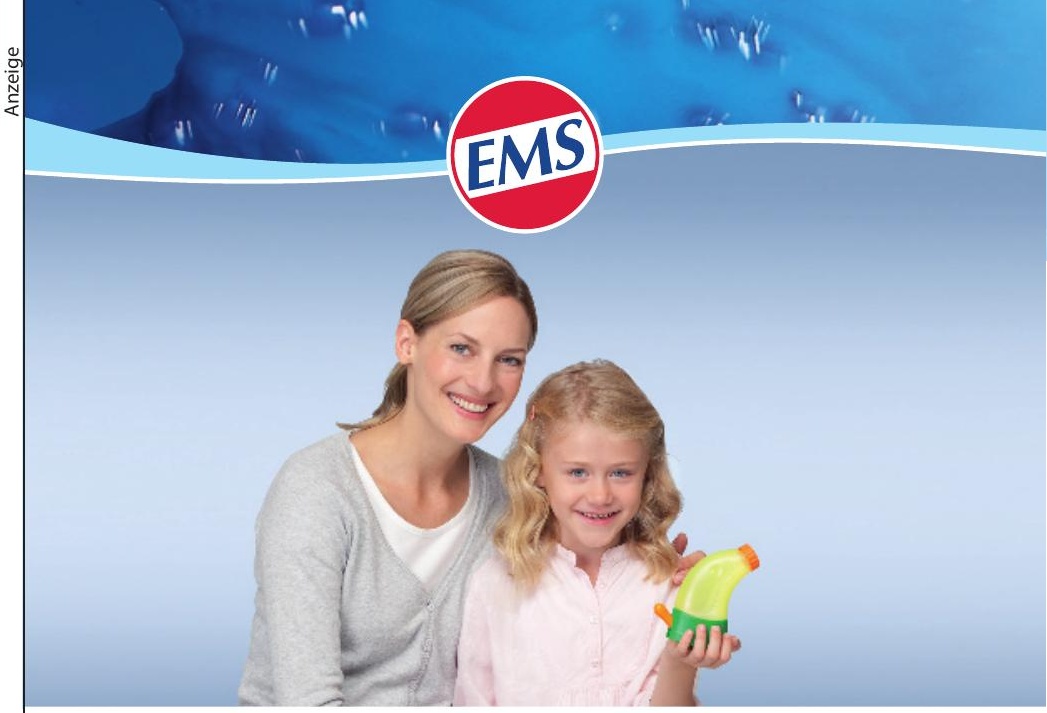

\section{Emser $^{\circledR}$ - Nasespülen jetzt kinderleicht!}

\section{Emser Salz ${ }^{\oplus}$ 1,475 g Kombipackung mit Applikationshilfe Emser ${ }^{\circledR}$ Kindernasendusche Nasanita $^{\circledR}$}

\section{Ihre Therapieempfehlung bei:}

- Rhinitiden, Rhinosinusitiden und Sinusitiden

- Adenoiden mit rezidivierenden Rhinitiden

- Paukenergüssen in Verbindung mit Rhinitiden

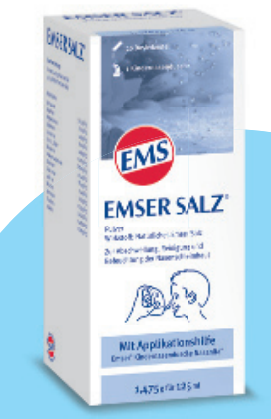

Emser Salz $1,475 \mathrm{~g}$

- lässt die Schleimhäute

sanft abschwellen

- löst aktiv festsitzenden Schleim

- wirkt auf rein natürlicher Basis

(a)

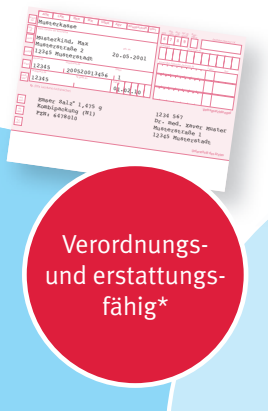

Speziell für Kinder entwickelt: Emser Kindernasendusche Nasanita ${ }^{\circ}$

- Angepasste Füllmenge (125ml)

- Kleines Nasenansatzstück

- Optimale Spüldruckregulierung durch integriertes Deckelventil

\footnotetext{
* bei gesetzlich Versicherten bis zum
} vollendeten 12. Lebensjahr www.kindernasendusche.de

\section{Jetzt die Fachinformation anfordern unter der Telefonnummer} 02603/9604-10 oder per Fax unter 02603/9604-36

Emser Salz ${ }^{\circ}$. Wirkstoff: Natürliches Emser Salz. Zusammensetzung: 100\% Natürliches Emser Salz (Analysedaten in $\mathrm{g} / \mathrm{kg}$ : $\mathrm{Li}^{+} 0,21 ; \mathrm{Na}^{+} 308,7 ; \mathrm{K}^{+} 6,11 ; \mathrm{Mg}^{2+} 0,291 ; \mathrm{Ca}^{2+} 0,016 ; \mathrm{Mn}^{2+}$

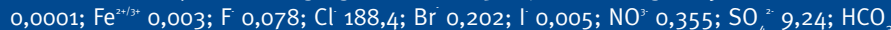
474,$4 ; \mathrm{CO}^{2}$ 14,0). Anwendungsgebiete: Zur unterstützenden Behandlung durch Befeuchtung, Reinigung und Abschwellung der Nasenschleimhaut bei banalen akuten Infektionen der oberen Atemwege und chronischen Rhinosinusitiden und als begleitende Behandlungsmaßnahme zur Beschleunigung des Heilungsprozesses nach endonasalen Nebenhöhlenoperationen. Gegenanzeigen: Rhinoliquorrhoe, stark erhöhte Neigung zu Epistaxis, relativ: Überempfindlichkeit der Atemwege, natrium- und kaliumarme Diät. Nebenwirkungen: gelegentlich leichte Reizerscheinungen wie Brennen und Kribbeln in der Nase und Kopfschmerzen, sehr selten Epistaxis. Siemens \& Co, 56119 Bad Ems 